\title{
EXAMINING THE BIAS FOR ORTHOGRAPHIC COMPONENTS USING AN APPARENT MOTION DETECTION TASK
}

\author{
Man-Ying WANG \\ Soochow University, Taiwan
}

\begin{abstract}
A novel task was used to examine how sublexical orthographic components are activated in the case of Chinese characters. Participants judged which of the two composing radicals of a horizontal character was displaced in an apparent motion (AM) detection task. The detection performance of semantic and phonetic radicals did not differ in Experiment 1. As additional recognition demands (naming or lexical decision) were imposed in Experiments 2 and 3, participants more efficiently detected the AM of the phonetic radical than the semantic radical when the character frequency was low. These findings demonstrate the usefulness of the AM detection task in probing radical activation. Implications for models of word and Chinese character recognition are also discussed.
\end{abstract}

Key words: sublexical processing, phonetic radical, semantic radical, word recognition, Chinese character

Prevalent models of visual word recognition emphasize the role of sublexical orthographic units in recognition regardless of the designation of recognition routes, the definition of orthographic units, or the type of script (Bowey, 1990; Seidenberg \& McClelland, 1989; Taft \& Forster, 1976; Zhou \& Marslen-Wilson, 1999a, b). In the case of Chinese, a logographic script, sub-lexical orthographic units may mediate recognition by reducing the ambiguity of the meaning or of the pronunciation of the whole word (Feldman \& Siok, 1999; Tan \& Perfetti, 1998). Some researchers consider the information that these orthographic components supply to be the primary source of constraint in initial lexical access (Tan \& Perfetti, 1998; Zhou, Shu, Bi, \& Shi, 1999). Despite the important role that orthographic units may play in supplying phonology and meaning, few studies have explored the early perceptual or attentional mechanism that activates these orthographic units. The lack of understanding of this mechanism may have contributed to some of the inconsistencies in research findings (cf. Liu, 2003). The nature of perceptual or attentional processing has escaped the focus of researchers partly due to the difficulty in devising an appropriate behavioral methodology that can adapt to the rapid recognition process without intruding upon it. This study devises an apparent motion detection task to probe the activation process for the orthographic components of Chinese characters. The question examined is whether there is any perceptual or attentional bias for particular orthographic components that alters as a function of recognition demands.

Correspondence concerning this article should be addressed to: Man-Ying Wang, Department of Psychology, Soochow University, 70 Lin-Shi Rd., Taipei, Taiwan (e-mail: mywang@scu.edu.tw) 
Chinese characters have an inherently hierarchical structure. Except for a few simple characters, the majority are compound characters composed of an array of components called radicals or bujians. Radicals are spatially distinct and re-occurring stroke patterns occupying semi-fixed locations in a character (Chen, 1996; Chen, Allport, \& Marshall, 1996; Lai \& Huang, 1997). There are a number of ways that radicals can be aligned within a character (Yeh, Li, \& Chen, 1997), among which the horizontal structure is the most common configuration (Gao \& Kao, 2002; Wang, 1998).

The majority of complex characters are phonetic characters composed of a semantic radical and a phonetic radical. The pronunciation of the phonetic radical is either exactly the same as or shares part of the pronunciation with the whole character in most phonetic compounds (Liu, Su, \& Chen, 2001; Zhou \& Marslen-Wilson, 1999b). As such, the phonetic radical has the potential to serve as a partially effective cue to character phonology which plays a critical role for many models of word recognition (Frost, 1998; Van Orden, Pennington, \& Stone, 1990). Semantic radicals, or bushous, are used extensively as indices in dictionary look-up. A semantic radical usually conveys the range or some aspect of the meaning of the whole character (Zhou \& Marslen-Wilson, 1999b) and thus has the potential to cue character meaning. Phonetic and semantic radicals are not only different in the function they serve, but also in their validity as a cue to the character's visual pattern. In general, the type frequency of phonetic radicals is lower than that of semantic radicals. That is, semantic radicals appear in many different characters but phonetic radicals appear in only a few (Li \& Chen, 1997). If the visual cueing value of a radical is defined as the probability of correctly deriving the whole character based on the knowledge that the radical is present, then this value should be generally higher in phonetic radicals than semantic radicals. As an example, the phonetic radical 司 (si1) appears in only five horizontal compound characters (飼 (si4, feed), 伺 (si4, wait), 嗣 (si4, offspring), 詞 ( $c i 2$, word), 祠 (ci2, temple)), while the semantic radical 言 (yan2) is part of 114 different horizontal characters. Consequently, the visual cueing value for the phonetic radical 司 (the probability of correctly guessing the character by the knowledge that 司 appears in its right half) would be $1 / 5$ while it is $1 / 114$ for the semantic radical 言 (the probability of correctly guessing the character by knowing 言 appears on the left side).

A third difference between phonetic and semantic radicals is that phonetic radicals can always stand alone as an independent character, while only some semantic radicals are stand-alone characters. Internally represented or meaningful patterns may attract attention (Christie \& Klein, 1995; Flowers \& Lohr, 1985; Flowers, Polansky, \& Kerl, 1981). Christie and Klein (1995), for example, presented a word and a non-word simultaneously at unpredictable locations and moved one of them after varying delays. Their participants were more efficient at discriminating the motion of words than of non-word items at short SOA (i.e., $100 \mathrm{~ms}$ ). Christie and Klein explained this finding in terms of attention being drawn to the location of the meaningful pattern (i.e., word).

Phonology cueing, high visual cueing, and meaningfulness support a speculation that phonetic radicals may enjoy a processing priority over semantic radicals in the visual recognition of Chinese characters. Nevertheless, it appears unlikely that the essential condition for the analysis of radical function, is accomplished at the early phases of word 
perception. Fortunately, a location regularity rule is present in the Chinese orthography. Semantic radicals are more frequently located at the left of a horizontal character or the top of a vertical character, and phonetic radicals tend to be located at the right or the bottom. Phonetic radicals are also slightly larger than semantic radicals in printed Chinese. If a visual recognition mechanism exploits the visual regularities present in the visual environment extensively (Marr, 1982), the correlation between these visual-spatial properties and the function of the radical may be used to distinguish between semantic and phonetic radicals,

In principle, there are two ways a phonetic radical may obtain processing priority. First, the reader may have a certain degree of attentional control over the radical activation process similar to people's flexible allocation of attentional resources to different levels of representation in hierarchical letters (Grice, Canham, \& Boroughs, 1983; Kinchla \& Wolfe, 1979; Martin, 1979; Navon, 1977; Pomerantz, 1983). Second, a phonetic radical and a semantic radical may be represented by different lexical sub-systems (Perfetti \& Tan, 1998, 1999) that result in different courses of activation. This difference may serve as the basis for a perceptual bias towards phonetic radicals. This study examines whether or not there is a bias for the phonetic radical, but can not distinguish between the two possible underlying mechanisms, though the discussed structural differences more directly imply the involvement of an attention allocation mechanism.

Past studies have not directly looked into the question of whether such a bias is indeed present. There is extensive evidence independently supporting the activation of phonetic radicals (Flores d'Arcais, Saito, \& Kawakami, 1995; Zhou \& Marslen-Wilson, 1999a, 1999b, also see Tan \& Perfetti, 1998 for a review) and semantic radicals (Feldman \& Siok, 1999; Leck, Weekes, \& Chen 1995; Zhou et al., 1999) in Chinese character recognition. One study addressed the issue of how semantic and phonetic radicals are selectively accessed. Chen and Allport (1995) manipulated task demands by changing the criteria for the matching of a pair of Chinese characters. In the pronunciation matching task, both the reaction times (RTs) and the error rates of different trials were higher when the target pair was a compound character with a phonetic radical (e.g., 怡 and 台) than when the target pair was a compound character with a semantic radical (e.g., 好 and 女). This pattern was reversed when the task required semantic matching. Chen and Allport considered these findings to result from a selective attention mechanism that can be directed according to the radical's function. Attention was selectively focused on the phonetic radical in the pronunciation task and on the semantic radical in the semantic task.

Other studies have examined the effects of radical function and location. Feldman and Siok (1997) manipulated radical function and location in a lexical decision task. The effect of type frequency was unambiguously found for phonetic radicals regardless of their location in the character, but not for semantic radicals. Chua (1999) asked participants to make same-different judgments on a pair of Chinese characters. The two characters always shared one radical and participants were informed of the location (left or right) of the redundant (shared) radical in advance. Chua found Chinese readers were not as successful in filtering out the redundant left radical as the right, while nonreaders did not demonstrate such asymmetry. This finding was interpreted as a difference in 
attention distribution that attention is drawn to radicals located on the right more than ones located on the left. However, since the stimuli used all had left semantic radicals and right phonetic radicals, the function of the radical could alternatively be the critical underlying dimension. In addition to Chua (1999), other studies have used lexical tasks and demonstrated the more important status of the right radical (Li \& Chen, 1999; Peng, Li, and Yang, 1997; Taft \& Zhu, 1997; Zhu \& Taft, 1994).

This study examines whether a performance advantage for the phonetic radical can be observed in an apparent motion (AM) detection task, and if so, what its relation is with radical location or character frequency. A horizontal compound character composed of two radicals is presented and the AM of one of the radicals is generated by displacing it towards the side, top, or bottom (see Fig. 1). The task of the participant is to indicate which of the two radicals moved. In the example shown in Fig. 1, following a fixation cross, 村 (cunl, village) is presented at the center of the screen for $100 \mathrm{~ms}$ (Frame 1). The radical 寸 (a phonetic radical located on the right) is displaced to the right, while the radical 木 stays at its original location to constitute Frame 2, which replaces Frame 1 and lasts for $16.7 \mathrm{~ms}$. 村 then reappears and the participant indicates whether it was the left or the right radical that moved. Although o produced a rightward movement in this example, three different directions of movements (i.e., up, down, and sideways) were divided evenly across trials.

The format of this task is somewhat similar to the Ternus task. In the Ternus task, three squares are horizontally aligned and two types of apparent motion are produced by repeatedly displacing the rightmost square laterally. A group movement (three squares are viewed as moving together) is perceived under long ISI (>50 ms) and element movement is perceived (the rightmost square moves laterally) for short ISI $(<50 \mathrm{~ms})$. The detection of element movement is suggested to depend on the parvocellular route, while the detection of group movement depends on the magnocellular route (Breitmeyer \& Ritter, 1986a, b; Petersik \& Pantle, 1989). A connection has been proposed between group movement perception and reading deficits (Cestnick \& Coltheart, 1999; Davis, Castles, McAnally, \& Gray, 2001). The AM detection task used in this study has a different focus from the Ternus task. It uses the perception of element movement to examine the presence of the hypothesized bias towards phonetic radicals.

It is assumed that the presence of any radical bias in Frame 1 can be probed by the offset of the radical followed by its onset at a peripheral location. Past studies have found stimulus offset and onset capture attention (Jonides \& Yantis, 1988; Yantis \& Gibson, 1994; Yantis \& Hillstrom, 1994; Yantis \& Johnson, 1990). It is thus reasonable to expect radical offset and onset not only to produce an AM, but also to drive the participant's attention to the "moved" radical. Since such attention capturing is not as effective when attention is already being directed elsewhere (Yantis \& Jonides, 1990), the attention allocated to radicals in Frame 1 is expected to affect subsequent attention capture by AM as well as AM detection performance. In other words, the ease of radical AM detection reflects the degree to which attention was directed to that radical in Frame 1. If a bias towards the phonetic radical is generated in Frame 1, then the AM of phonetic radicals would be easier to detect than the AM of semantic radicals. Either the lack of any 


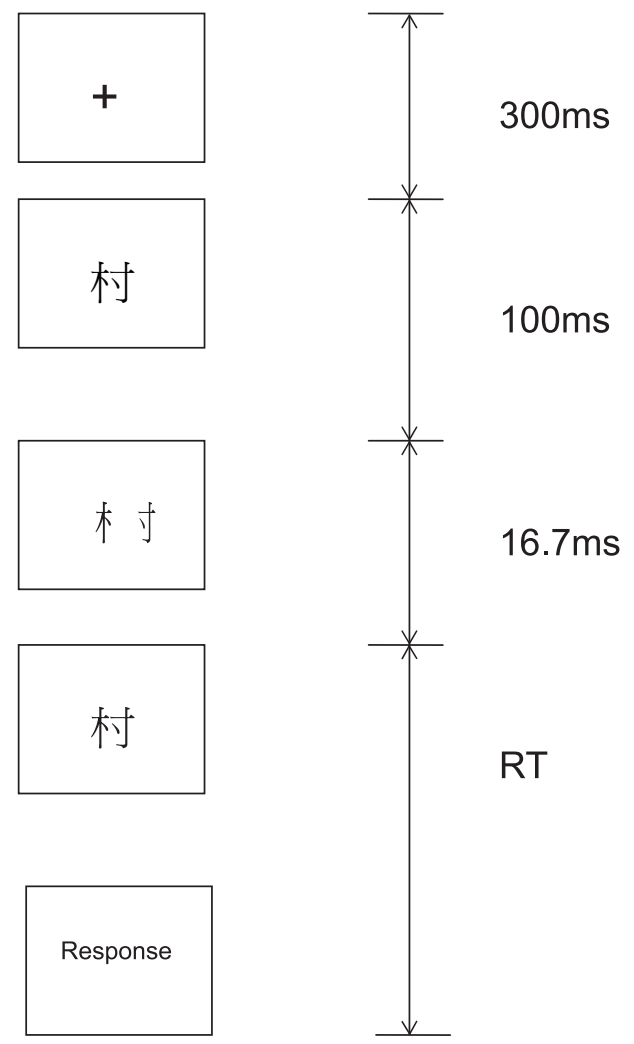

Fig. 1. The presentation sequence in the apparent motion detection task.

difference in AM detection performance between phonetic and semantic radicals or a better detection performance for semantic radicals would constitute counter-evidence for the hypothesis of a processing bias for phonetic radicals.

The designation of this task represents an attempt to reduce task-specific strategies in the study of Chinese character perception. For example, in the same-different matching task adopted by Chen and Allport (1995), an attention allocation strategy specific to their task may have been induced. Since the matching response was explicitly requested to be based on pronunciation or meaning, participants could have employed their explicit orthographic knowledge (that phonetic radicals help cue pronunciation and semantic radicals help cue meaning) and deliberately allocated their attention as such. The resultant attention allocation pattern is not as interesting since its generalizability is questionable. In contrast, since the AM detection task is not lexical in nature, orthographic knowledge is irrelevant to the task. Attention allocation or the radical activation pattern reflected in the AM detection performance is unlikely susceptible to task-specific strategies.

In this study, three experiments are used to examine the effect of radical function on AM detection performance. The effect of radical location is also tested since the attentional selection of the phonetic radical is likely a spatially based mechanism 
(Downing \& Pinker, 1985; Tsal, 1983) that reflects location regularity in Chinese orthography. If the hypothesized bias towards the phonetic radical is generated by selecting the spatial location highly associated with its presence, then one would expect an interaction between radical function and location so that the phonetic radical advantage is observed only when it is located on the right. It is also possible that the selection is driven by size differences between these two types of radicals. In this latter case, there would not be any effect of location. Character frequency and radical frequency are also manipulated in all experiments since past studies have demonstrated that they may interact with the activation of orthographic components in both English (Allen \& Madden, 1990; Drewnowski \& Healy, 1977; Healy \& Drewnowski, 1983; Johnson, Allen, \& Strand, 1989) and Chinese (Chen, 1986; Wang, 2002). Radical token frequency (i.e., radical frequency weighed by the frequency of the character in which the radical is present), rather than radical type frequency, is examined here because the former reflects the familiarity of the radical to the participant (Fang \& Wu, 1989; Li \& Chen, 1997) and supposedly plays a role in the radical activation process (cf. Masuda \& Saito, 1999).

A completely crossed design for all four variables using horizontal Chinese characters, though preferred, is not de facto possible. There are only a few characters consisting of left phonetic radicals with high radical frequency or of right semantic radicals with low radical frequency, regardless of the character frequency. In order to achieve as much power to examine the relevant effects as possible, a supplement list was formed in addition to the experimental list of items. The experimental list contained an orthogonal manipulation of radical function, radical location, and character frequency (eight conditions). The supplement list was composed of four conditions in order to examine the independent effect of radical frequency in combination with some of the conditions from the experimental list. Another control list was composed of digits and served as the baseline for the effect of location.

These three lists were mixed altogether in one experimental session. Participants detected the AM of these items in Experiment 1. They made additional naming or lexical decision responses in Experiments 2 and 3. Recognition demands were imposed in Experiments 2 and 3 to guarantee lexical access when participants performed the AM detection task. It is possible that attention allocation was contingent on task demands, that is, whether the stimulus shared a feature property relevant to performing the task (Folk, Remington, \& Wright, 1994). Radical function (and/or location) would not be a relevant task attribute and was unlikely to direct attention if participants in Experiment 1 simply treated the character as a non-lexical visual pattern. Since the three experiments of this study differed only slightly, one methods section is used to describe them all.

\section{METHOD}

\section{Participants:}

In each experiment, 30 undergraduate students at Soochow University who are native speakers of Mandarin served as participants. They were each paid NT\$100 (U.S. \$3.00) for their participation in the experiment. 


\section{Design and Stimuli:}

The stimuli used were the same across all three experiments. The character and radical token frequency were computed according to the Mandarin Promotion Council's (1998) character frequency norms. Radical function (semantic vs. phonetic), radical location (left vs. right), and character frequency (low vs. high) were manipulated to form eight conditions in the main experimental list, with 12 characters in each condition (see the Appendix for a layout of the design and examples). The supplement list was composed of four conditions (also see the Appendix) with a mean radical frequency matching those for right semantic radicals in the experimental list. By combining data from the experimental list and the supplement list, it was possible to at least partially test whether radical function has an effect when radical token frequency is matched, as well as whether radical token frequency itself affects performance. The control list was composed of double-digit numerals (e.g., 58). The location of the moved digit was manipulated to create two conditions. Results from the control list may help clarify the relationship of the location effect (if any) with handedness. Instead of balancing for the hand of response, the control list was used since the pursued effect was expected to be small and likely to be washed out when half of the responses were based on the nonpreferred hand.

The direction to which a radical component or digit moved was randomly decided for each participant and counterbalanced across participants so that $1 / 3$ of the trials in each condition moved to the top, $1 / 3$ to the bottom, and $1 / 3$ laterally. A total of 168 trials (i.e., $12 \times(8+4+2)=168$ ) were generated for each participant and randomly presented. Efforts were also made to keep the repetition of radicals (across characters) to a minimum - the average number of repetitions of the target radical ranged from 1 to 1.85 across conditions. Additional pseudo-characters were used in Experiment 3. The design for the pseudocharacter was intended to be radical function $(2) \times$ radical location $(2) \times$ radical frequency $(2)$. However, one of the eight conditions was not fulfilled. Pseudo-characters with low-frequency semantic radicals located on the right were not created since their inclusion would have unbalanced the mean radical repetition. There were thus seven corresponding conditions for pseudo-characters with 12 items in each cell with a total of 84 items. An additional 36 pseudo-characters were generated as fillers (in half of which the left radical moved in the AM detection task and the other half the right radical moved) to balance the proportion of "yes" and "no" trials in the lexical decision task. Each participant thus saw a total of 288 trials $(168+84+36)$ in Experiment 3. For all three experiments, 24 practice trials were generated using materials not repeating but are representative of the experimental stimuli.

All stimuli were presented in black at the center of an otherwise white background using the standard Kai font. At a viewing distance of $85 \mathrm{~cm}$, the fixation cross, the character, and the radical subtended a visual angle of approximately $0.88^{\circ} \times 0.88^{\circ}, 1.6^{\circ} \times 1.6^{\circ}$ and $0.8^{\circ} \times 1.6^{\circ}$. The double-digit numeral and each digit were of about the same size as the character and the radical.

\section{Procedure:}

The experiment was administered in a quiet, dim room. The presentation and timing of the stimuli as well as data collection were controlled by an AMD Athlon 800 PC using the DMDX experimental software. Stimuli were presented on a Viewsonic G773 17" color monitor. The timing and sequence of stimulus presentation was as follows: fixation $(300 \mathrm{~ms})$, character or numeral $(100 \mathrm{~ms})$, radicals in separation $(16.7$ $\mathrm{ms}$ ), character (stayed until response) (see Fig. 1). At of the start of the last character frame, response time was measured and the next trial began $3000 \mathrm{~ms}$ afterwards no matter whether a response was made or not. Participants pressed the "F" or "J" key on the keyboard to indicate whether it was the left or the right component that had moved.

Participants were informed that the characters or numerals presented on the screen consisted of two parts and that one of them would move to the top, bottom, or side. No matter which direction the radical or the digit moved, the participant's task was to indicate whether the left or the right component moved by pressing the appropriate key. They were asked to perform the task as quickly and as accurately as possible. Participants in Experiment 1 performed the AM detection task, while participants in Experiments 2 and 3 were requested to make an additional naming or lexical decision response on the character that had just been presented for the AM judgment. In each trial of Experiment 2, as soon as the detection response was made, the computer screen turned dark and a beep immediately sounded as the cue for the participant to name the character or the double-digit numeral. No matter whether the participant made the naming response or not, the next trial started $2000 \mathrm{~ms}$ after the start of the beep. In Experiment 3, the character display in the AM detection task was replaced by two characters: "real" and "pseudo" (from right to left). The participant was 
asked to press the right key if the stimulus was a character or a numeral, and press the left key if the stimulus was a pseudo-character. Similarly, no matter whether a response was made or not, the next trial started 2000 ms afterwards.

\section{RESULTS AND DISCUSSION}

RTs beyond three $S D$ 's of each participant's mean or lower than $200 \mathrm{~ms}$ were considered extreme. Extreme and error trials were excluded from the RT analysis. Across the three experiments, average error rates were low $(1.4 \%, 1.3 \%$, and $1.7 \%)$, as were the proportions of extreme trials $(1.4 \%, 2.5 \%$, and $1.9 \%)$. Also excluded from the RT analysis in Experiments 2 and 3 were $5.7 \%$ and $10.7 \%$ of total trials in which participants were correct at AM detection but erred on the subsequent naming or lexical decision task. These trials were excluded to ensure all detection data came from trials in which participants had processed the character to the lexical level. Although naming or lexical decision errors were excluded from the RT analysis, they were not treated as detection errors in the error-rate ANOVA. Error rate data was arcsine-transformed before the ANOVA. Effects are reported as reliable only if they were significant in both the subject and item analyses.

\section{Experiment 1}

A character frequency $(2) \times$ radical function $(2) \times$ radical location $(2)$ three-way ANOVA was performed on the RT and error-rate data of the experimental list. Participants were faster at detecting AM in right radicals than left ones $(F 1(1,29)=7.47$, $p<.05 ; F 2(1,88)=15.81, p<.0005)$ by $8.5 \mathrm{~ms}$. They also made more errors on phonetic than semantic radicals $(F 1(1,29)=5.81, p<.05 ; F 2(1,88)=5.04, p<.05)(1.94 \%$ vs. $0.97 \%$ ) (see Fig. 2). An ANCOVA was used to rule out the role of radical frequency since it was not balanced across conditions. To select among potential covariates, data from the experimental list were pooled with the supplement list in computing the correlation between radical frequency and item mean RT's. This correlation was $-0.10(p>.20)$ for radical token frequency and $0.08(p>.30)$ for radical type frequency (combinability). A logarithmic transformation of radical token frequency increased its correlation with RT's to -0.15 ( $p=.058)$, but no similar transformation could be used to increase the correlation between radical type frequency and RTs. Analysis of covariance was thus performed on the data of the experimental list using logarithmic token frequency as the covariate. In this analysis, radical location was still a significant effect, but somewhat less so ( F2) 1 , $87)=5.91, p<.05)$. Since the complexity (number of strokes) of the moved radical also appeared slightly unbalanced, its correlation with the RTs was computed $(-0.13, p>.10)$. The number of strokes was added as the second covariate and the ANCOVA showed that the location effect remained $(F 2(1,87)=4.63, p<.05)$. This ANCOVA also indicated that the error rate effect of radical function became unreliable $(F 2(1,87)=1.00, p>.30)$ and radical function remained unreliable in the RT data (see Fig. 3 for the adjusted mean RTs). There was no other significant effect. As a whole, the only effect that remains reliable after adjusting for radical token frequency and radical complexity is radical 


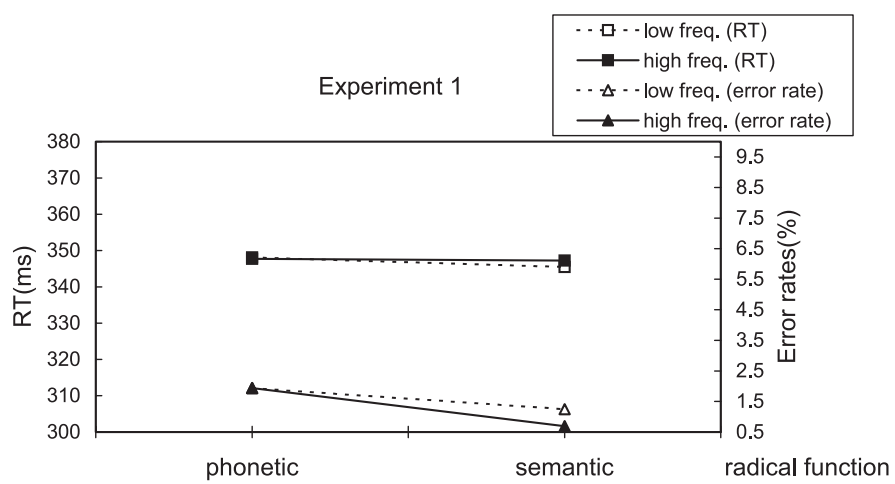

Experiment 2

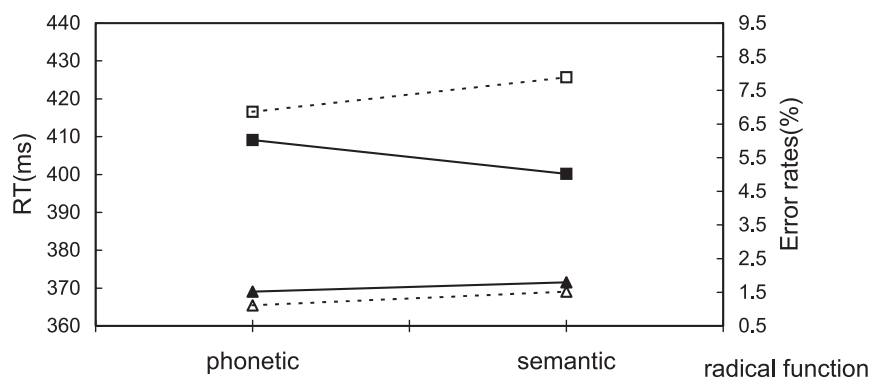

Experiment 3

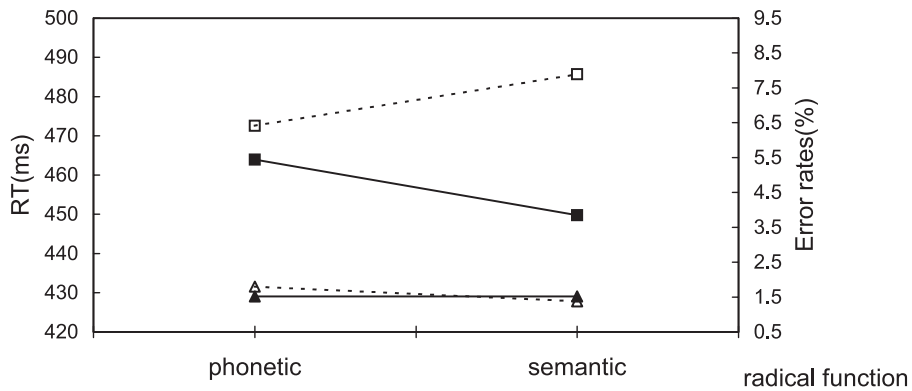

Fig. 2. Apparent motion detection RT's and error rates as a function of character frequency and radical function for Experiment 1 (detection only), Experiment 2 (detection + naming) and Experiment 3 $($ detection + LDT).

location. The right is faster than the left by $6.2 \mathrm{~ms}$ in their adjusted means.

To evaluate the independent role of radical frequency, four conditions from the experimental list were pooled with the supplement list to form a balanced design: character frequency $(2) \times$ radical frequency $(2) \times$ group (2). The two conditions of the group variable were "phonetic radicals on the right" vs. "semantic radicals on the left". Although this group variable did not encompass all possible combinations of radical function and location, it did correspond with location regularity and thus should be 


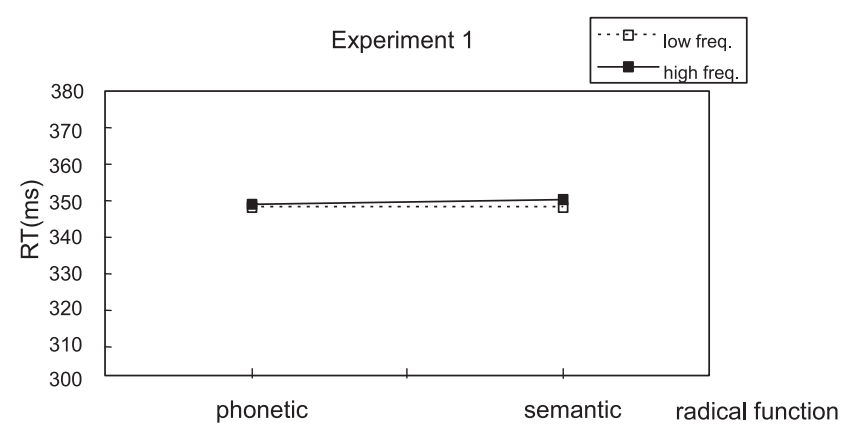

Experiment 2

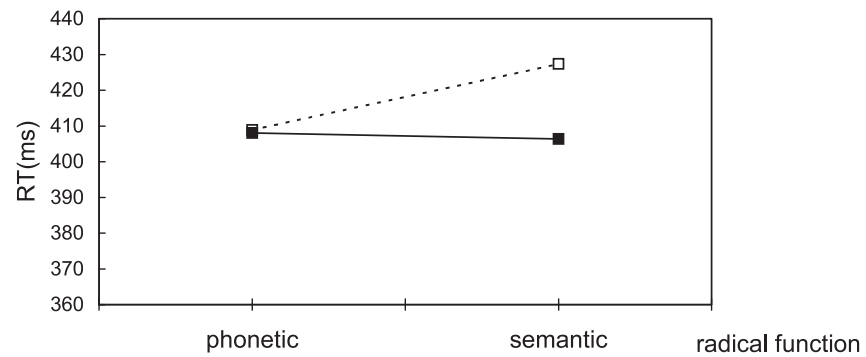

Experiment 3

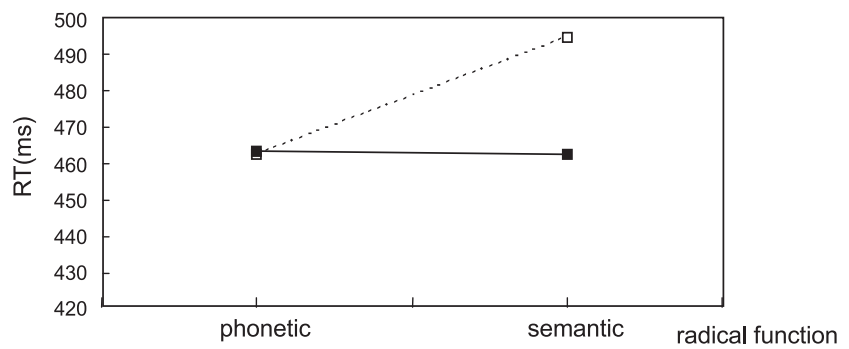

Fig. 3. The adjusted mean RT's (adjusted for radical token frequency and the number of strokes in the radical) as a function of character frequency and radical function for Experiment 1 (detection only), Experiment 2 (detection + naming) and Experiment 3 (detection + LDT).

representative of a considerable proportion of Chinese characters. The ANOVA indicated that radical frequency itself did not exhibit any effect (both $F \mathrm{~s}<1$ ) either in the RT or the error rate analysis. An interaction was found between character frequency, radical frequency, and group in the RT data $(F 1(1,29)=9.29, p<.005 ; F 2(1,88)=7.05$, $p<.01)$. Low-frequency characters were also faster than high-frequency ones $(F 1(1$, $29)=5.83, p<.05 ; F 2(1,88)=6.39, p<.05)$. Right phonetic radicals were slightly faster than left semantic radicals $(F 1(1,29)=3.52, .05<p<.10 ; F 2(1,88)=9.96, p<.005)$. There was not any other effect in either the RT or the error rate analysis.

Finally, the analysis of the control list (i.e., double-digit numerals) showed reliable 
location effects. The AM detection for digits located on the right was $17 \mathrm{~ms}$ faster than ones on the left $(F 1(1,29)=25.79, p<.0001 ; F 2(1,22)=22.39, p<.0001)$, with no difference in error rates.

Radical function did not exhibit any reliable effects in Experiment 1 after correcting for the effect of radical frequency using ANCOVA. On the other hand, the effect of location (an advantage of the right radical) cannot be accounted for by radical frequency. This radical location effect could be attributable to handedness since a "right" advantage also turned up in numerals (the control list). Although radical complexity was slightly unbalanced across conditions, none of the effects was altered by including it as a covariate. As a whole, the AM detection performance in Experiment 1 was not meaningfully affected by character frequency, radical function, or radical location, and thus does not support the hypothesis of a processing priority for the phonetic radical.

However, we cannot rule out the possibility that participants in Experiment 1 were treating the two-radical character stimulus as a two-component array, as with the doubledigit numerals. Experiments 2 and 3 thus impose recognition demands (i.e., naming and lexical decision, respectively) to guarantee lexical processing of the character.

\section{Experiment 2}

Analysis of the experimental list showed that participants spent $17 \mathrm{~ms}$ more to process low-frequency characters than high frequency ones $(F 1(1,29)=13.5, p<.001$; $F 2(1,87)=13.83, p<.005)$. More interesting, there was an effect of radical function in the form of an interaction with character frequency $(F 1(1,29)=5.93, p<.05 ; F 2(1$, $87)=5.03, p<.05$ ) (see Fig. 2). Detecting the motion of the phonetic radical was faster by $14 \mathrm{~ms}$ for low-frequency characters, while it was the semantic radical that was faster by $9 \mathrm{~ms}$ when the character frequency was high. This interaction was not diminished by considering the variation in radical token frequency since it remained significant $(F(1$, $86)=6.64, p<.05)$ in the ANCOVA using the logarithmic radical token frequency as the covariate. The effect of character frequency was also significant $(F(1,86)=6.30, p<.05)$ in the ANCOVA. These two effects were reliable even when the number of strokes was added as a second covariate $(F(1,86)=6.40$ for the character frequency $\mathrm{x}$ radical function interaction, and $F(1,86)=6.08$ for the character frequency effect, both $p$ 's $<.05)$. The detection of phonetic radicals was faster than semantic radicals only when character frequency was low $(p<.05)$ and they were no different from each other for highfrequency characters $(p>.8)$ (see Fig. 3 for adjusted means). The hypothesis of a phonetic radical advantage is supported when it pertains to low-frequency characters.

Although the location effect was not significant in the RT data, it was marginally reliable in the subject analysis $(F 1(1,29)=3.56, .05<p<.10)$ and significant in the item analysis $(F 2(1,88)=5.97, p<.05)$ for the error rate data. Participants made $1.18 \%$ more errors for radicals located on the right. However, the significant item effect became marginal in the ANCOVA either when the logarithmic radical token frequency was used as the sole covariate $(F(1,87)=3.49, .05<p<.10)$ or when it and the number of strokes both served as covariates $(F(1,86)=2.91, .05<p<.10)$.

Part of the experimental list was again pooled with the supplement list in order to 
examine the effect of radical frequency. Unlike Experiment 1, radical frequency itself had an effect on error rates $(F 1(1,29)=5.96, p<.05 ; F 2(1,87)=4.85, p<.05)$. The error rate was higher in low-frequency radicals $(1.67 \%$ vs. $0.83 \%)$. Character frequency again affected reaction times $(F 1(1,29)=6.01, p<.05 ; F 2(1,88)=7.47, p<.01)$. Highfrequency characters were $11 \mathrm{~ms}$ faster than low-frequency characters. There was no other significant effect in the RT or the error-rate analysis.

The only reliable effect in the analysis of the control list was the one for location in error rates - participants made more errors for digits located on the right $(F 1(1$, $29)=4.01, .05<p<.06 ; F 2(1,22)=6.19, p<.05)(2.22 \%$ vs. $0.28 \%)$.

Imposing a demand to name the character indeed modified the manner in which participants processed it as reflected in their AM detection performance. They spent more time to process low-frequency characters (than high-frequency ones) presumably due to the difficulty in accessing phonology for low-frequency characters. On the other hand, participants in Experiment 1 did not appear to treat the character as a lexical item and even spent slightly more time on high-frequency characters than low-frequency ones. The recognition demands in Experiment 2 successfully induced a change in participants' mode of character processing and resulted in the interaction effect between radical function and character frequency.

The hypothesis of a phonetic radical advantage was verified for characters of low but not high frequency. In fact, there was a tendency for the semantic radical to be processed faster than the phonetic radical in the latter case (see Fig. 2). However, this tendency may be attributed to the fact that semantic radicals are generally higher in token frequency (and thus more familiar) than phonetic radicals since this effect diminished in the ANCOVA using radical frequency as the covariate (see Fig. 3). The slight variation in the complexity of the radical (in terms of number of strokes) across conditions again was not related to any of the effects found.

Before concluding a bias towards phonetic radicals exists in the lexical processing of low-frequency Chinese characters, it is necessary to replicate the findings of Experiment 2 by imposing a different recognition demand (i.e., lexical decision) in Experiment 3.

\section{Experiment 3}

The major findings of Experiment 2 were replicated. Low-frequency characters took $22 \mathrm{~ms}$ longer to process $(F 1(1,29)=17.06, p<.0005 ; F 2(1,88)=20.35, p<.0001)$. Character frequency also interacted with radical function $(F 1(1,29)=5.34, p<.05 ; F 2(1$, $88)=7.14, p<.01$ ) (see Fig. 2). Phonetic radicals were faster than semantic radicals by $13 \mathrm{~ms}$ when the character frequency was low, while semantic radicals were faster by 14 $\mathrm{ms}$ when the character frequency was high. The detection of radicals located on the right was faster and more accurate than that of left radicals (RT: $F 1(1,29)=3.20, .05<p<.10$; $F 2(1,88)=8.38, p<.005$. Error rate: $F 1(1,29)=6.45, p<.05 ; F 2(1,88)=8.12$, $p<.01)$. In the analysis of covariance using the number of strokes and logarithmic radical token frequency as covariates, the effect of character frequency and the interaction between character frequency and radical function continued to be reliable $(F(1$, $86)=8.16, p<.01$ and $F(1,86)=11.32, p<.005$, respectively). Phonetic radicals were 
faster than semantic radicals by $32 \mathrm{~ms}$ in their adjusted means for low-frequency characters $(p<.005)$, while semantic radicals were no different from phonetic radicals $(p>.8)$ for high-frequency characters (see Fig. 3). The reaction time effect for radical location found in the ANOVA may be related to the variation in radical frequency between locations since it became unreliable using radical frequency as the covariate $(F<1)$, but remained significant when the number of strokes was the covariate $(F(1$, $86)=9.05, p<.005)$. The location effect on error rates remained significant when logarithmic radical frequency served as the covariate by itself $(F(1,86)=5.88, p<.05)$ or conjointly with the number of strokes $(F(1,86)=5.89, p<.05)$.

Pseudo-characters were divided into three groups-left/low radical frequency, left/ high radical frequency, and right/high radical frequency, with half of the items in each group being phonetic radicals and the other half semantic radicals. The radical function (2) $\times$ group (3) ANOVA did not uncover any significant effect in reaction time, but the effect of radical function was significant in the error rate analysis $(F 1(1,29)=5.18$, $p<.05 ; F 2(1,66)=5.35, p<.05)$. Participants made fewer errors on phonetic radicals than semantic radicals $(0.7 \%$ vs. $1.9 \%)$.

Data of the experimental and supplement list were pooled in a manner similar to earlier experiments for a radical frequency $\times$ character frequency $\times$ group ANOVA. In addition to the effect of character frequency $(F 1(1,29)=4.37, p<.05 ; F 2(1,88)=9.39$, $p<.005)$ in reaction times, group was the only significant effect. Phonetic radicals located on the right were $21 \mathrm{~ms}$ faster $(F 1(1,29)=8.18, p<.01 ; \mathrm{F} 2(1,88)=13.24$, $p<.0005)$ and $1.25 \%$ more accurate $(F 1(1,29)=4.08, .05<p<.06 ; F 2(1,88)=8.52$, $p<.005)$ than semantic radicals located on the left.

Finally, the control list (numerals) was analyzed separately and the effect of location was significant-detecting the motion of digits located on the right was $63 \mathrm{~ms}$ faster $(F 1(1$, $29)=47.52, p<.0001 ; F 2(1,22)=73.38, p<.0001)$ and $6.4 \%$ more accurate $(F 1(1$, $29)=12.76, p<.005 ; F 2(1,22)=40.36, p<.0001)$ than digits located on the left.

A phonetic radical advantage in AM detection was found for low-frequency characters when either naming (Experiment 2) or lexical decision (Experiment 3) was the demanded recognition response for the character. Both Experiments 2 and 3 found a slower reaction time for low-frequency characters relative to high-frequency characters, illustrating a difficulty in accessing the phonology or judging the lexicality for lowfrequency characters.

Pseudo-characters can be regarded as characters of "very low frequency" and the results of pseudo-characters should somehow resemble those for low-frequency characters. In support of this explanation, Experiment 3 indeed found a consistent effect of radical function in pseudo-characters, though in the error rate data only. Participants were more efficient in detecting the AM of phonetic radicals in both low-frequency characters (faster reaction time) and pseudo-characters (fewer errors).

Although radical token frequency is considered to be a potential determinant of participants' detection performance, it did not render a clear effect across the three experiments. Its logarithmic transformation, however, exhibited reliable correlation with detection RTs in Experiments 2 and 3. It accounts for the slight advantage of the semantic 
radical in high-frequency characters (compare Fig. 2 and 3), indicating that this slight advantage may have emerged from the greater activation of these semantic radicals with higher average token frequency (Masuda \& Saito, 1999). The phonetic radical advantage in low-frequency characters would then appear to have been underestimated since the phonetic radical suffers from being low in token frequency.

The effect of radical location was not consistent across experiments. Experiment 3 found an advantage for radicals located on the right in both RT's and error rates. Experiment 1 found a similar effect only in reaction times, while Experiment 2 revealed an error-rate advantage for the left, instead of the right (radicals and numerals). The comparison between radicals and numerals was, however, consistent. The effects of location for numerals always emerged in the same measure and in the same direction as those for radicals, though more sizable. There appeared to be experiment-wise location biases that resulted in similar effects for radicals and numerals. Handedness alone could not interpret these effects since Experiment 2 actually exhibited a slight advantage towards the left. The reason underlying these effects is not yet clear.

\section{GENERAL DISCUSSION}

Is it true that a bias for the phonetic radical is generated as one tries to recognize a Chinese character? The results of the three experiments provide the following answers: (1) When specific recognition demands are not imposed and participants treat the character as a two-component array, there is no evidence of a priority setting for any type of radical (Experiment 1). (2) When participants are requested either to name or to decide on the lexical status of the character and if the recognition is difficult (low character frequency), the phonetic radical is processed more efficiently than the semantic radical in an AM detection task (Experiments 2 and 3).

The importance of these findings is two-fold. On the one hand, they demonstrate that the AM detection task is a useful tool for probing the selection or activation of orthographic components. The finding of a bias towards the phonetic radical in lowfrequency characters, though novel, is consistent with previous studies in which radical effects were mediated by character frequency. Among them, the regularity effect refers to the phenomenon that compound characters having the same pronunciation as their phonetic radicals (i.e., regular) are named faster (or RTs are faster in lexical decisions, in some cases) than frequency-matched simple characters (Seidenberg, 1985). This regularity effect, however, is restricted to low-frequency characters (Hue, 1992). In a similar vein, radical priming effects also rely on character frequency. Zhou and MarslenWilson (1999a, b) indicated that the phonetic radical located in a low-frequency character may be automatically decomposed and prime the naming of a semantically related target character. Conversely, the naming of a low-frequency target character is slowed by a prime character that is semantically related to the phonetic radical contained in the target. In both cases, the character containing the phonetic radical that is decomposed and responsible for the priming effect was chosen to be of low frequency in Zhou and 
Marslen-Wilson (1999a, b). When character frequency is explicitly manipulated, as in Ding, Peng and Taft (2004), the radical prime produces a priming effect in the lexical decision time of the target character, but only when the character frequency is low. Although Ding et al. did not manipulate radical function, it is likely that their radical primes were predominantly phonetic radicals since they were all stand-alone characters. These studies and current study consistently found radical-level effects reliable only when the character frequency is low, suggesting that the AM detection task does indeed probe into a process that's active in character recognition.

How should findings of this study be interpreted? It appears that the most compatible theoretical delineation would be the dual-route model of word recognition (Coltheart, 1978; Coltheart, Curtis, Atkins, \& Haller, 1993) which accounts for the difference between high- and low-frequency characters in terms of different recognition routes. Although high-frequency characters may follow the lexical route to check for an entry in the mental lexicon, low-frequency characters are processed by a non-lexical route and the GPC rule is applied to generate pronunciation. The reader selectively relies on the phonetic radical as a partially effective cue to the pronunciation of the whole character in the process of grapheme-to-phoneme transformation. This explanation fits with the findings of Experiment 2 and would also predict a phonetic radical advantage in the lexical decision task (Experiment 3) if accessed phonology is used as the indication of lexicality.

One might question this explanation by pointing out that the phonetic radical is not very effective in serving as a cue to character phonology (Feldman \& Siok, 1999). According to Liu et al. (2001), the percentage of traditional Chinese characters whose pronunciation is the same as their phonetic radicals is $29 \%$ (at most $37 \%$ if one uses the number of compound characters as the denominator). This percentage increases to $39 \%$ for simplified characters due to some structural differences between traditional and simplified characters. It thus does not seem to be adaptive and is probably detrimental to recognition by maintaining a mechanism that is affected by component properties. In addition to the finding that viewers do respond to low-validity cues in standard attention paradigms (Erikson \& Yeh, 1985), these calculations do not take character frequency into account. It is not known whether any previous studies have separately computed the percentage of regular characters for high- and low-frequency characters. As long as the percentage of regular characters is reasonably high among low-frequency characters, current findings are not too surprising.

In addition to the dual-route account that assumes an active role for the reader in selectively setting a processing priority for the phonetic radical when the character frequency is low, the current findings can also be interpreted in terms of the results of a race between the character and the component channel of processing (Huang \& Wang, 1992; Wang, 2002). The radical wins the race with the character channel only when the character frequency is low (Chen, 1986; Drewnowski \& Healy, 1977; Wang, 2002). As such, the phonetic radical is activated more readily than the semantic radical probably because it is always internally represented (a stand-alone character) (Ding et al., 2004).

The interactive constituency model (Perfetti \& Tan, 1998, 1999; Tan \& Perfetti, 
1997) requires additional assumptions to interpret the findings of this study. This model follows the basic tenet of the interactive activation model (McClelland \& Rumelhart, 1981) and assumes that Chinese characters are hierarchically represented and interactively processed, but in multiple lexicons or subsystems, instead of one. Characters, including semantic and phonetic radicals that are stand-alone characters, are represented in the character orthographic lexicon. The noncharacter orthographic lexicon organizes radical components that are not stand-alone characters. Since the phonetic radical generally resides in a different lexicon from the semantic radical (i.e., the character orthographic lexicon vs. the noncharacter lexicon), it is possible for this model to accommodate the current findings (i.e., by assuming that the mapping to the phonological and meaning subsystems from the noncharacer lexicon is not present or generally weaker than that from the character orthographic lexicon). The phonetic radical advantage may result from the feedback facilitation from the phonological and/or meaning subsystem that is stronger than that for the semantic radical. When character frequency is high, mapping to the phonological or the meaning subsystems from the character is sufficiently fast to surpass that of radicals, allowing no room for any effect of radicals.

The multilevel interactive-activation model of Chinese character recognition (Taft, Liu, \& Zhu, 1999; Taft \& Zhu, 1997; Zhu \&Taft, 1994) cannot provide a reasonable explanation for the current findings without additional assumptions. This model also adopts the interactive-activation framework, but it assumes that radicals are processed in a left-to-right manner in the building up of character activation. Properties of the right radical (the slower in processing) thus constrain character processing (Taft \& Zhu, 1997). This model would also have to assume that radical activation is largely inhibited when character frequency is high. Similarly, it would also have to make some additional assumptions to accommodate the radical function effect (e.g., interactions between attention capture by the phonetic radical and the left-to-right processing order).

Although different models may accommodate the interaction effect between radical function and character frequency, they vary with respect to the assumed source of the phonetic radical advantage. In the dual-route account, the phonetic radical advantage results from a true priority setting that would have to be modulated by some kind of selective attention mechanism (e.g., Folk et al., 1994). Other models rely on the relative speed of processing of radical components and the whole character as well as feedback activation to explain the phonetic radical advantage. There is no active or deliberate priority setting in these latter cases. Results from this study, of course, can not distinguish between them, though the dual-route account more readily explains the effect of task demand (i.e., Experiment 1 vs. Experiments 2 \& 3).

Regardless of the theoretical position, these accounts of a phonetic radical advantage in terms of an attentional or perceptual bias supply a specific reason for the involvement of left middle frontal area whose activation is unique to the Chinese logograph (relative to alphabetic languages) (Tan et al., 2000; Tan et al., 2001). Peak activation was observed in the left prefrontal area (BA 9 and 46) when the task was generation (Tan et al., 2000), semantic relatedness judgment, or homophone judgment (Tan et al., 2001). The above discussion suggests a working memory component for any task of Chinese character 
recognition. The left prefrontal areas are related to Chinese logograph reading either because they help modulate attention allocation to the phonetic radicals when whole character matching is difficult (the dual-route account), or due to their roles in maintaining the radical and character representation in the process of recognition (the race and interactive constituency account). This conjecture is consistent with the attribution of the specific roles of these areas to executive control, spatial working memory, and the maintenance and manipulation of representations (Collette \& Van der Linden, 2002; Courtney, Petit, Maisog, Ungerleider, \& Haxby, et al., 1998; Wagner, Maril, Bjork \& Schacter, 2001).

To conclude, this study presents evidence for the usefulness of the apparent motion detection task in probing the activation of orthographic components during recognition. A bias towards the phonetic radical in Chinese character recognition was found when the character frequency was low. Although novel, this finding is consistent with the existing literature in the sublexical processing of the Chinese characters. These findings also serve as additional evidence constraining model-building for Chinese character recognition.

\section{REFERENCES}

Allen, P. A., \& Madden, D. J. 1990. Evidence for a parallel input serial analysis model of word processing. Journal of Experimental Psychology: Human Perception \& Performance, 16, 48-64.

Bowey, A. J. 1990. Orthographic onsets and rimes as functional units of reading. Memory \& Cognition, 18, 419-427.

Breitmeyer, B. G., \& Ritter, A. 1986a. The role of visual pattern persistence in bistable stroboscopic motion. Vision Research, 26, 1801-1806.

Breitmeyer, B. G., \& Ritter, A. 1986b. Visual persistence and the effect of eccentric viewing, element size and frame duration on bistable stroboscopic motion percepts. Perception \& Psychophysics, 39, 275280 .

Cestnick, L., \& Coltheart, M. 1999. The relationship between language-processing and visual-processing deficits in developmental dyslexia. Cognition, 71, 231-255.

Chen, H. C. 1986. Component detection in reading Chinese characters. In H. S. R. Kao and R. Hoosain (Eds.), Linguistics, psychology, and the Chinese language (pp. 1-10). Hong Kong: Center for Asian Studies, University of Hong Kong Press.

Chen, H. C. 1996. The analysis of the bujian structural frequency for common Chinese characters. Paper presented at the 1996 annual convention of the Chinese Psychological Association, Taipei.

Chen, Y. P., \& Allport, D. A. 1995. Attention and lexical decomposition in Chinese word recognition: Conjunctions of form and position guide selective attention. Visual Cognition, 2, 235-268.

Chen, Y. P., Allport, D. A., \& Marshall, J. C. 1996. What are the functional orthographic units in Chinese word recognition: The stroke or stroke pattern? The Quarterly Journal of Experimental Psychology, 49A, 1024-1043.

Christie, J., \& Klein, R. 1995. Familiarity and attention: Does what we know affect what we notice? Memory \& Cognition, 23(5), 547-550.

Chua, F. K. 1999. Visual perception of the Chinese character: configural or separable processing. Psychologia, 42, 209-221.

Coltheart, M. 1978. Lexical access in simple reading tasks. In G. Underwood (Ed.), Strategies of information processing (pp. 151-216). San Diego, CA: Academic Press.

Coltheart, M., Curtis, B., Atkins, P., \& Haller, M. 1993. Model of reading aloud: dual-route and paralleldistributed-processing approaches. Psychological Review, 100, 589-608.

Collette, F., \& Van der Linden, M. 2002. Brain imaging of the central executive component of working 
memory. Neuroscience and Biobehavioral Reviews, 26, 105-125.

Courtney, S. M., Petit, L., Maisog, J. M., Ungerleider, L. G., \& Haxby, J. V. 1998. An area specialized for spatial working memory in human frontal cortex. Science, 279, 1347-1351.

Davis, C., Castles, A., McAnally, K., \& Gray, J. 2001. Lapses of concentration and dyslexic performance on the Ternus task. Cognition, 81, B21-B31.

Ding, G., Peng, D., \& Taft, M. 2004. The nature of the mental representation of radicals in Chinese: a priming study. Journal of Experimental Psychology: Learning, Memory, \& Cognition, 2, 530-539.

Downing, C. J., \& Pinker, S. 1985. The spatial structure of visual attention. In M. I. Posner \& O. S. M. Marin (Eds.), Attention and performance XI (pp. 171-187). Hillsdale, NJ: Lawrence Erlbaum Associates Inc.

Drewnowski, A., \& Healy, A. F. 1977. Detection errors on the and_and: Evidence for reading units larger than the word. Memory \& Cognition, 5, 636-647.

Eriksen, C. W., \& Yeh, Y. Y. 1985. Allocation of attention in the visual field. Journal of Experimental Psychology: Human Perception \& Performance, 11, 583-597.

Fang, S. P., \& Wu, P. 1989. Illusory conjunctions in the perception of Chinese characters. Journal of Experimental Psychology: Human Perception \& Performance, 15, 434-447.

Feldman, L. B., \& Siok, W. W. T. 1997. The role of component function in visual recognition of Chinese characters. Journal of Experimental Psychology: Learning, Memory, \& Cognition, 23, 776-781.

Feldman, L. B., \& Siok, W. W. T. 1999. Semantic radicals contribute to the visual identification of Chinese characters. Journal of Memory and Language, 40, 559-576.

Flores d'Arcais, G.. B., Saito, H., \& Kawakami, M. 1995. Phonological and semantic activation in reading kanji characters. Journal of Experimental Psychology: Learning, Memory, \& Cognition, 21, 34-42.

Flowers, J. H. \& Lohr, D. J. 1985. How does familiarity affect visual search for letter strings? Perception \& Psychophysics, 37, 557-567.

Flowers, J. H., Polansky , M. L., \& Kerl, S. 1981. Familiarity, redundancy, and the spatial control of visual attention. Journal of Experimental Psychology: Human Perception \& Performance, 7, 157-166.

Folk, C. L., Remington, R. W., \& Wright, J. H. 1994. The Structure of attentional control: Contingent attentional capture by apparent motion, abrupt onset, and color. Journal of Experimental Psychology: Human Perception \& Performance, 20, 317-329.

Frost, R. 1998. Towards a strong phonological theory of visual word recognition: true issues and false trails. Psychological Bulletin, 123, 71-99.

Gao, D. G., \& Kao, H. S. R. (2002). Psycho-geometric analysis of commonly used Chinese characters. In H. S. R. Kao, C. K. Leong, \& D. G. Gao (Eds.,) Cognitive neuroscience studies of the Chinese language (pp. 195-206). Hong Kong: Hong Kong University Press.

Grice, G. R., Canham, L., \& Boroughs, J. M. 1983. Forest before trees? It depends where you look. Perception \& Psychophysics, 33, 121-128.

Healy, A. F., \& Drewnowski, A. 1983. Investigating the boundaries of reading units: Letter detection in misspelled words. Journal of Experimental Psychology: Human Perception \& Performance, 9, 413426.

Huang, J. T., \& Wang, M. Y. 1992. From unit to gestalt: Perceptual dynamics in recognizing Chinese characters. In H. C. Chen, \& O. J. L.Tzeng, (Eds.), Language processing in Chinese (pp. 3-35). NorthHolland: Elsevier Science Publishers.

Hue, C. W. 1992. Recognition processing in character naming. In H.-C. Chen \& O. J. L. Tzeng (Eds.), Language processing in Chinese (pp. 93-107). Amsterdam: North-Holland.

Johnson, N. F., Allen, P. A., \& Strand, T. L. 1989. On the role of word frequency in the detection of component letters. Memory \& Cognition, 17, 474-482.

Jonides, J., \& Yantis, S. 1988. Uniqueness of abrupt visual onset in capturing attention. Perception \& Psychophysics, 43, 346-354.

Kinchla, R. A., \& Wolfe, J. M. 1979. The order of visual processing: “Top-down”, "bottom-up", or "middleout". Perception \& Psychophysics, 25, 225-231.

Lai, C., \& Huang, L. T. 1997. A comparative study concerning the frequency counts of Chinese character constituents. Paper presented at the International Symposium on Cognitive Processes of the Chinese Language. Hong Kong. 
Leck, K. J., Weekes, B. S., \& Chen, M. J. 1995. Visual and phonological pathways to the lexicon: Evidence from Chinese readers. Memory \& Cognition, 23, 468-476.

Li, H., \& Chen, H. C. 1997. Processing of radicals in Chinese character recognition. In H. C. Chen (Ed.), Cognitive processing of Chinese and related Asian languages (pp. 141-160). Hong Kong: The Chinese University Press.

Li, H., \& Chen, H. C. 1999. Radical processing in Chinese character recognition: Evidence from lexical decision. Psychologia, 42, 199-208.

Liu, I. M. 2003. Introduction to Chinese character/word processing. Chinese Journal of Psychology, 45, 1-9.

Liu, I. M., Su, Y. R., \& Chen, S. C. 2001. The phonetic function of Chinese phonetic radicals. Kaohsiung, Taiwan: Fu-Weng.

Mandarin Promotion Council. 1998. A survey report on the often-used words and phrases in 1997. Taipei, Taiwan: Ministry of Education.

Marr, D. 1982. Vision. San Francisco: Freeman.

Martin, M. 1979. Local and global processing: The role of sparsity. Memory \& Cognition, 7, 476-484.

Masuda, H., \& Saito, H. 1999. Two types of radical frequency effects on Japanese Kanji character recognition. Psychologia, 42, 222-242.

McClelland, J. L., \& Rumelhart, D. E. 1981. An interactive activation model of context effects in letter perception: Part 1, An account of basic findings. Psychological Review, 88, 375-407.

Navon, D. 1977. Forest before trees: The precedence of global features in visual perception. Cognitive Psychology, 9, 353-383.

Peng, D. L., Li, Y. P., \& Yang, H. 1997. Orthographic processing in the identification of Chinese characters. In H. C. Chen (Ed.), Cognitive processing of Chinese and related Asian languages (pp. 85-108). Hong Kong: The Chinese University Press.

Perfetti, C. A., \& Tan, L. H. 1998. The time course of graphemic, phonological, and semantic activation in visual Chinese character identification. Journal of Experimental Psychology: Learning, Memory, and Cognition, 24, 101-118.

Perfetti, C. A., \& Tan, L. H. 1999. The constituency model of Chinese word identification. In J. Wang, A. W. Inhoff, \& H. C. Chen (Eds.), Reading Chinese script: A cognitive analysis (pp. 115-134). Mahwah, NJ, US: Lawrence Erlbaum Associates.

Petersik, A., \& Pantle, T. 1989. The two-process distinction in apparent movement. Psychological Bulletin, 106, 107-127.

Pomerantz, J. R. 1983. Global and local precedence: Selective attention in form and motion perception. Journal of Experimental Psychology: General, 112, 516-540.

Seidenberg, M. S. 1985. The time course of phonological code activation in two writing systems. Cognition, 19, 1-30.

Seidenberg, M. S., \& McClelland, J. L. 1989. A distributed, developmental model of visual word recognition and naming. Psychological Review, 96, 523-568.

Taft, M., \& Forster, K. I. 1976. Lexical storage and retrieval of polymorphemic and polysyllabic words. Journal of Verbal Learning \& Verbal Behavior, 15, 607-620.

Taft, M., \& Zhu, X. 1997. Sub-morphemic processing in reading Chinese. Journal of Experimental Psychology: Learning, Memory, \& Cognition, 23, 761-775.

Taft, M., Liu, Y., \& Zhu, X. 1999. Morphemic processing in reading Chinese. In J. Wang, A. W. Inhoff, \& H. C. Chen (Eds.), Reading Chinese script: A cognitive analysis (pp. 91-113). Mahwah, NJ, US: Lawrence Erlbaum Associates.

Tan, L. H., \& Perfetti, C. A. 1997. Visual Chinese character recognition: Does phonological information mediate access to meaning? Journal of Memory \& Language, 37, 41-57.

Tan, L. H., \& Perfetti, C. A. 1998. Phonological codes as early sources of constraint in Chinese word identification: A review of current discoveries and theoretical accounts. Reading and Writing, 10, 165-200.

Tan, L. H., Lin, H. L., Perfetti, C. A., Spinks, J. A., Fox, P. T., \& Gao, J. H. 2001. The neural system underlying Chinese logograph reading. NeuroImage, 13, 836-846.

Tan, L. H., Spinks, J. A., Gao, J. H., Liu, H. L., Perfetti, C. A., Xiiog, J., Stofer, K. A., Pu, Y., Liu Y., \& Fox, P. T. 2000. Brain activation in the processing of Chinese characters and words: A functional MRI 
study. Human Brain Mapping, 10, 16-27.

Tsal, Y. 1983. Movements of attention across the visual field. Journal of Experimental Psychology: Human Perception \& Performance, 9, 523-530.

Van Orden, G. C., Pennington, B. F., \& Stone, G. O. 1990. Word identification in reading and the promise of subsymbolic psycholinguistics. Psychological Review, 97, 488-522.

Wagner, A. D., Maril, A., Bjork, R. A., \& Schacter, D. L. 2001. Prefrontal contributions to executive control: fMRI evidence for functional distinctions within lateral prefrontal cortex. Neuroimage, 14, 13371347.

Wang, M. Y. 1998. The orthographic processing in the visual recognition of Chinese characters. NSC-892413-H-031-003.

Wang, M. Y. 2002. The nature of character-component interaction in Chinese character perception. Psychologia, 45, 162-175.

Yantis, S., \& Gibson, B. S. 1994. Object continuity in apparent motion and attention. Canadian Journal of Psychology, 48, 182-204.

Yantis, S., \& Hillstrom, A. P. 1994. Stimulus-driven attentional capture: Evidence from equiluminant visual objects. Journal of Experimental Psychology: Human Perception \& Performance, 20, 95-107.

Yantis, S., \& Johnson, D. N. 1990. Mechanisms of attentional priority. Journal of Experimental Psychology: Human Perception \& Performance, 16, 812-825.

Yantis, S., \& Jonides, J. 1990. Abrupt visual onsets and selective attention: Voluntary versus automatic allocation. Journal of Experimental Psychology: Human Perception \& Performance, 16, 121-134.

Yeh, S. L., Li, J. L., \& Chen, I. P. 1997. The perceptual dimensions underlying the classification of the shapes of Chinese characters. Chinese Journal of Psychology, 39, 47-74.

Zhou, X., \& Marslen-Wilson, M. 1999a. The nature of sublexical processing in reading Chinese characters. Journal of Experimental Psychology: Learning, Memory, \& Cognition, 25, 819-837.

Zhou, X. \& Marslen-Wilson, M. 1999b. Sublexical processing in reading Chinese. In J. Wang, A. W. Inhoff \& H. C. Chen (Eds.), Reading Chinese script - A cognitive analysis (pp. 37-63). Mahwah, NJ, US: Lawrence Earlbaum Associates.

Zhou, X., Shu, H., Bi, Y., \& Shi, D. 1999. Is there phonologically mediated access to lexical semantics in reading Chinese? In J. Wang., A. W. Inhoff., \& H. C. Chen (Eds.), Reading Chinese script: A cognitive analysis (pp. 135-171). Mahwah, NJ, US: Lawrence Erlbaum Associates.

Zhu, X., \& Taft, M. 1994. The influence of perceptual experience on Chinese character processing. In H. W. Chang, J. T. Huang, C. W. Hue and O. J. L. Tzeng (Eds.), Advances in the study of Chinese language processing (pp. 85-99). Taipei, Taiwan: Department of Psychology, National Taiwan University. 
Appendix

Mean character frequency, radical frequency, and the number of strokes in the experimental list and the supplement list

\begin{tabular}{|c|c|c|c|c|c|c|}
\hline $\begin{array}{l}\text { Character } \\
\text { frequency }\end{array}$ & Radical type & $\begin{array}{l}\text { Radical } \\
\text { location }\end{array}$ & $\begin{array}{l}{ }^{\mathrm{a}} \text { Mean } \\
\text { character } \\
\text { frequency }\end{array}$ & $\begin{array}{c}{ }^{\text {a Mean }} \\
\text { radical } \\
\text { frequency }\end{array}$ & $\begin{array}{c}\text { Mean } \\
\text { number of } \\
\text { strokes }\end{array}$ & Examples \\
\hline \multicolumn{7}{|c|}{ The experimental list } \\
\hline Low & Phonetic & Left & 7 & 79 & 8.7 & 煩 (jia2) \\
\hline Low & Phonetic & Right & 8 & 69 & 6.6 & 仟 (qianl) \\
\hline Low & Semantic & Left & 7 & 71 & 8.2 & 耘 (yun2) \\
\hline Low & Semantic & Right & 9 & 4520 & 4.5 & 朔 (shuo4) \\
\hline High & Phonetic & Left & 86 & 93 & 7.1 & 雅 (ya3) \\
\hline High & Phonetic & Right & 80 & 96 & 7.6 & 洞 (dong4) \\
\hline High & Semantic & Left & 87 & 180 & 6.6 & 幼 $($ you 4$)$ \\
\hline High & Semantic & Right & 88 & 4793 & 4.2 & 雕 (diaol) \\
\hline \multicolumn{7}{|c|}{ The supplement list } \\
\hline Low & Phonetic & Right & 7 & 3789 & 8 & 駱 (luo4) \\
\hline Low & Semantic & Left & 6 & 4081 & 7 & 酮 (tong2) \\
\hline High & Phonetic & Right & 83 & 5378 & 6 & 彼 (bi3) \\
\hline High & Semantic & Left & 87 & 4655 & 5 & 旺 (wang4) \\
\hline
\end{tabular}

a The reported frequency is based on count per million characters (Mandarin Promotion Council, 1998). 